\title{
Mini-review: recent updates on the mathematical modelling of radiation-induced bystander effects
}

\author{
Muhamad Hanis Nasir, Fuaada Mohd Siam* \\ Department of Mathematical Science, Faculty of Science, Universiti Teknologi Malaysia, 81310 UTM Johor Bahru, Johor, Malaysia \\ *Corresponding author: fuaada@utm.my
}

Article history

Received 17 February 2017

Accepted 21 April 2017

\begin{abstract}
Radiotherapy treatment uses ionizing radiation (IR) in order to kill cancer cells. However, the IR exerted its effects outside the radiation field and causes cell death in healthy cells. This effect namely as radiation-induced bystander effects (RIBE) phenomenon. The scope of the overview of the RIBE phenomenon discussed in this paper includes the RIBE mechanism, danger signaling process, deoxyribonucleic acid (DNA) double-strand breaks (DSBs) damage and the damage repair. This paper extended with the discussion of several mathematical models used to describe the RIBE phenomenon. The discussions towards the mathematical models include the models of signals concentration, the models of bystander effects and the survival fraction model. Mathematical modelling and computer simulation are powerful tools used to understand the biological phenomenon of RIBE. The suitable mathematical model of repair and mis-repair DNA DSBs damage has been briefly reviewed in view of the relevance of this model towards RIBE phenomenon. The outcome of this paper suggested recommendations for future research on the suitable mathematical model and simulation analysis in describing the complexity of RIBE phenomenon.
\end{abstract}

Keywords: RIBE phenomenon, DNA DSBs damage, bystander effects, cell survival fraction

\section{INTRODUCTION}

Apart from the effects of DNA damage in cells traversed by ionizing radiation (IR), nearby cells that do not traverse by IR also have subsequent damage effects. Initially, it has been thought that only cells traversed by IR have subsequent damage. This dogma had been changed after findings proved that there are damage effects such as genetic and biochemical alterations observed in the nearby cells and distant cells that do not traverse directly by IR [1-2]. This phenomenon has been referred as the radiation-induced bystander effects (RIBE) [3] This phenomenon gives us a new thought on understanding towards how IR exerts its effects. Other than RIBE, effects of post-radiation also include adaptive response, abscopal effects and genomic instability [45].

RIBE is a phenomenon observed in the bystander cells which are not directly traversed by IR. This phenomenon has been examined experimentally by many researchers for over more than two decades. This phenomenon happened when the irradiated cells produced damaging signal molecules to non-irradiated cells which are not traversed directly by IR ([5-8] and references therein). The bystander cells show many biological effects such as single-strand breaks (SSBs), double-strand breaks (DSBs), chromosomal aberrations, sister chromatid exchange, carcinogenesis, micronucleation, increased the frequency of apoptosis, reduced clonogenic efficiency, oncogenic transformation and DNA repair delays [4, 6-7, 9-12]. It is known that DNA damage in directly traversed cells is the main target of IR but it is now accepted that the final outcome of the IR not restricted to targeted area of radiation only. There are researchers proposed the possible mechanism of RIBE. RIBE is a complex mechanism and till today, the actual mechanism underlying the RIBE is still open for debated. However, it is not our intention to discuss the overall mechanism of RIBE here. Interested reader may refer to $[6-7,10]$ for intensive discussions of RIBE.

\section{OVERVIEW OF MECHANISMS OF RIBE}

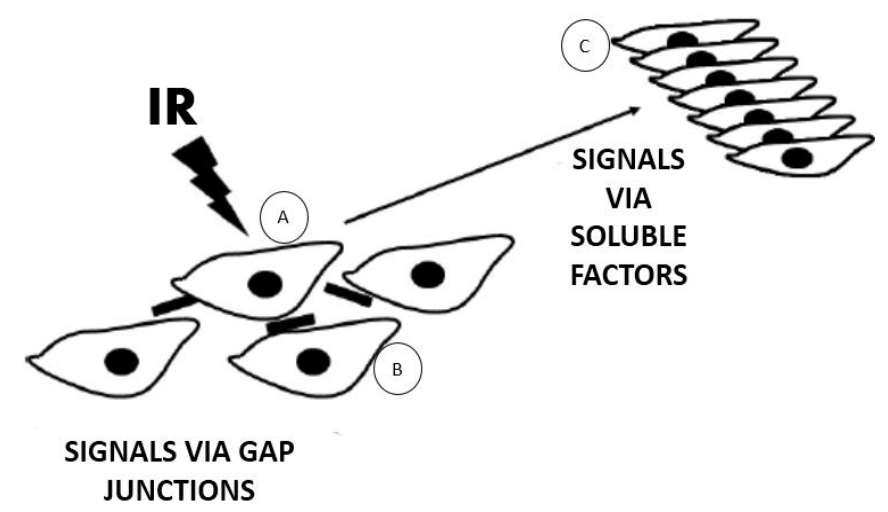

Fig. 1 Illustration of signals released from the irradiated cells by Baskar [2].

Baskar [2] had proposed the schematic representation of RIBE phenomenon. Cell A is the cell that traversed directly by IR, cell B is the bystander (adjacent) cell and cell $\mathrm{C}$ is the distant cells or tissue. After cell A had been traversed by IR, DSBs will form as an oxidative DNA lesion and cell A become the irradiated cells. According to Han and $\mathrm{Yu}$ [13], bystander signal molecules are produced very quickly (less than 2.5 minutes) from irradiated cells after radiation process. The RIBE effects happen in cell $\mathrm{B}$ because of signaling process via gap junction intercellular communication (GJIC), while cell $\mathrm{C}$ becomes affected because of signaling process via soluble factors released into the cultured medium, that is distant-cell signaling intercellular communication (DSIC). 
There are many kind of danger signal molecules produced by the irradiated cells. The candidates of the danger signal molecules may include reactive oxygen species (ROS), interleukin-8 (IL8), nitric oxide (NO), interleukin-1 (IL1), reactive nitrogen species (RNS) and interleukin-6 (IL6) [1, 5-6, 14]. Najafi et al. [7] elaborated more about short life and long life category of signal molecules. Oxygen and nitrogen free radicals are short-living signals which can affect only at adjacent cells while long-living signals (e.g.: hydrogen peroxide) can travel long distances and causing DNA damage at distant cells.

Cells can communicate with other cells through GJIC and DSIC. The content of a cell is surrounded by a thin envelope called cell membrane. This surface of cell controls the passage dissolved substances into and out of the cell through the membrane receptors. GJIC can transfer the danger signal molecules from an irradiated cell to a non-irradiated cell and hence producing many impacts of cellular damage effects [10]. The contribution of GJIC and DSIC communication have strong impacts in cultured cells where the danger signal molecules allowed to interact with other cells through passive diffusion or with receptors on the cell's membrane. Desouky et al. [1] also mentioned that the propagation of bystander effects can be observed in several studied using $\alpha$-particle, $\beta$-particle and $\gamma$-rays which the process involves the interaction of intercellular cell communication.

\section{Experimental evidence}

There are experiments which are related to the phenomenon of radiation-induced bystander effects have been done. A review-article by Nikitaki et al. [8] listed many experimental findings on RIBE mechanism which is observed in various systems; including human, rodents, fish and plants (Table 1 in [8]). The earliest bystander effects of radiation has been determined by Mole [15] in 1953 where there are actions involved at distant cells within the same organism. In 1992, Nagasawa and Little [16] revealed that there is a connection between the irradiated cells and non-irradiated cells. The result showed the damage effects on $30 \%$ of cells is sister chromatid exchanges when only $1 \%$ of cells being radiated with $\alpha$-particle. This is because of the response of non-irradiated cells toward danger signal molecules released in the medium through the pathway of intercellular cell communication.

Experiment by Sokolov et al. [17] in year 2005 using micro-beam facility showed there is induction of $\gamma-\mathrm{H} 2 \mathrm{AX}$ foci in the bystander cells after radiation process. In the same year, $\mathrm{Hu}$ et al. [18] also reported that after $\alpha$-particle traversed $1 / 10$ of the cells nuclei, there is excessive $\gamma-\mathrm{H} 2 \mathrm{AX}$ immunofluorescence observed in bystander cells. Another experiment using micro-beam facility reported by Han and $\mathrm{Yu}$ [13] claims that only $1 \%$ of the cells population survived after $\alpha$-particle was traversed directly towards $20 \%$ of the cells population.

Besides micro-beam facility method, medium transfer method has been conducted. As reported by Sasaki et al. [19], individual V79 cells were irradiated with carbon K-shell X-ray micro beam and then the V79 cells were located in the petri dish that containing non-irradiated cells. Seymour and Mothersill [20] in year 2000 justified that the danger signal molecules released by the irradiated cells and then affecting nonirradiated cells in the cultured medium. Yang et al. [21] showed that there are $\gamma-\mathrm{H} 2 \mathrm{AX}$ foci formation in the bystander cells after being in cultured with cells that had been radiated with X-rays radiation. The trans-well insert culture dish had been used in order to demonstrate the danger signal molecules released into the medium.

The classification of experiments is divided into two categories, either in vivo or in vitro experiment. In vivo refers to experiments conducted within the living organism, while in vitro refers to experiments conducted within the glass. Interested reader may refer to Hatzi et al. (Table 1 in [22]) and Widel (Table 1 in [5]) for the summary of experimental works related to RIBE in vivo and in vitro, respectively.

\section{DNA DAMAGE AND REPAIR PROCESS}

Damage caused by ionizing radiation can be categorized in two types, targeted and non-targeted radiation damage. Double-strand breaks (DSBs) are considered as the critical lesions induced by ionizing radiation [23-24]. In non-targeted radiation damage, some suggestions made by researchers $[1-2,4-5,25]$ pointed out that the damage experienced by non-targeted cells after being in contact with danger signal molecules is the same (so-called "mimic") as the targeted cells damage by ionizing radiation. These bystanding cells respond to signals emitted by the irradiated cells and in turn behave as if they have been directly affected by ionizing radiation $[11,26]$. So here are the types of DNA damages and repair process will be discussed.

\section{A direct biomarkers of DSBs}

$\gamma-\mathrm{H} 2 \mathrm{AX}$ foci is a phosphorylated form of histone H2AX which induced after the formation of DSBs and it is an important response towards DNA damage. Several researchers $[6,13-14,27]$ agreed that $\gamma-$ $\mathrm{H} 2 \mathrm{AX}$ foci formation is acted as a biomarker of DSBs. Many experimental results observed that $\gamma-\mathrm{H} 2 \mathrm{AX}$ foci are formed in bystander non-irradiated cells which leads to the agreement that DNA DSBs damage formed in the bystander cells. Wang et al. [6] also mentioned that each discrete $\gamma$-H2AX foci contains a single DNA DSBs damage and number of DSBs can be measured by counting the number of $\gamma-\mathrm{H} 2 \mathrm{AX}$ foci in the cell's nucleus.

\section{Types of DNA damage}
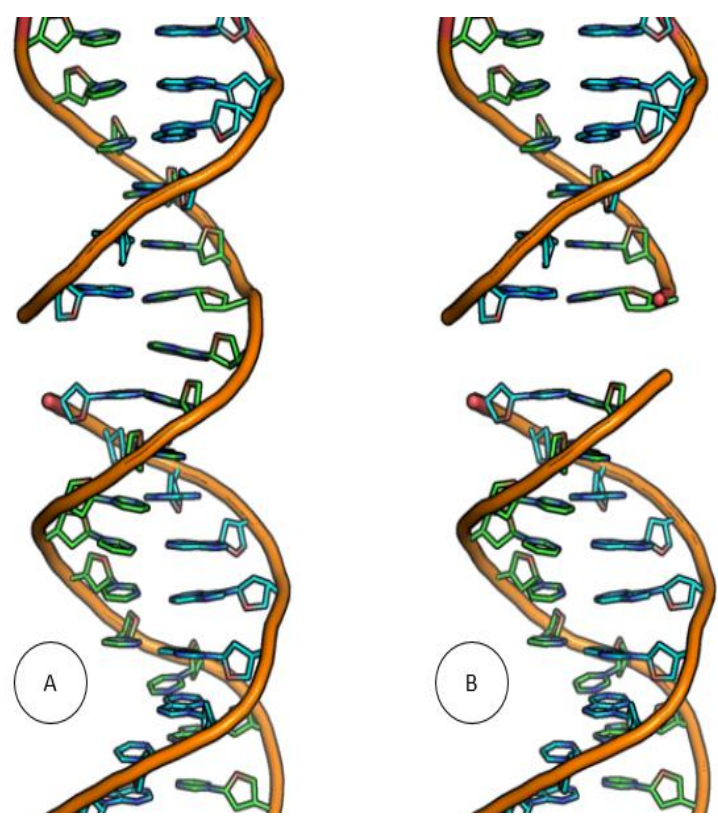

Fig. 2 (A) Illustration of DNA SSB and (B) illustration of DNA DSB.

Fig. 2(A) shows the single-strand break (SSB) while Fig. 2(B) is double-strand break (DSB) (break on both opposite sides). Among all the biological effects of RIBE, DSBs are considered as the insidious initial lesions of DNA damage and also considered as the simplest form representing the complex DNA damage $[6,14,28]$. SSBs are not considered harmful because they are repaired using the opposite strand, while DSBs effect will lead to chromosome damage which then can cause cell death. The interaction of two DSBs can result in cell death, chromosome aberrations, carcinogenesis and mutation [23].

\section{DNA damage repair mechanisms}

The processes of DSBs repair in a mammalian cell are categorized into two types, homologous recombination repair (HRR) and nonhomologous end joining (NHEJ) [23-24]. Both processes cannot be performed simultaneously; the specific repair process performed by the damaged cell depends on the phase of the cell cycle. For more details about the cell cycle phases in HRR and NHEJ, see page 62 in [23].

Homologous recombination repair (HRR) is a template-assisted repair which this pocess requires identical or nearly identical undamaged DNA strand as a template (Fig. 3(A)). HRR is a common pathway used in eukaryotes cells and it is an error-free process because the repair process is performed by copying the undamaged homologous chromosome or chromatid. In contrast, non-homologous end joining (NHEJ) is an end-to-end joining repair where the break ends are directly 
ligated without the need for a homologous template (Fig. 3(B)). The processes of NHEJ involve ends recognition, end processing, fill-in-synthesis and ends ligation. NHEJ is the dominant repair mechanism in many mammalian cells and it is an error-prone process. Any interested reader can refer to [23-24, 28] for more details about the process.

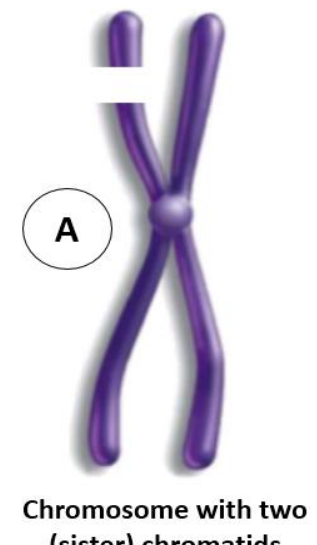

(sister) chromatids

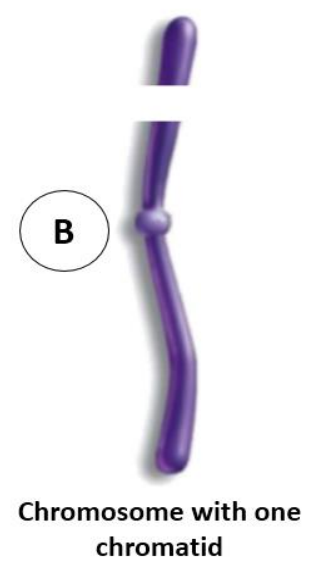

chromatid

\section{Fig. 3 (A) Illustration of HRR and (B) NHEJ repair processes.}

Although cells have two complex repair mechanisms for dealing with DSBs, some of the damage are not repaired and are mis-repaired [29]. These remaining DSBs and mis-repaired DSBs will lead to chromosome aberration, increased of sister chromatid exchanges, micronucleic formation and cell death $[9,30]$. The repair process in RIBE also experienced a repair delay effect $[11,31]$.

Another important factors which can be activated after the formation of DSBs is the phosphorylation of ataxia-telengiectasia mutated kinase (ATM). ATM phosphorylated several proteins in order to activate cell cycle chekpoints, DNA repair and apoptosis. Ojima et al. [32] studied the repair kinetics of DSBs by investigating the ATM foci in bystander MRC- 5 cells co-cultured with X-ray irradiated cells. The ATM foci could be observed 0.05 hour after irradiation in bystander cells and remain steady for almost 48 hours. On contrary, DSBs induced by direct radiation were repaired relatively quick [6, 32].

\section{MODELS OF SIGNAL CONCENTRATION}

As discussed earlier, the DNA damage in bystander cells is because of the danger signal molecules released by the irradiated cells. In the experiment, the signals released by irradiated cells are transferred everywhere in the petri dish [31]. The diffusion of the bystander signals follows the Brownian motion with the mean square displacement $(r)$ at a time, $t$ is given as:

$$
\left\langle r^{2}(t)>=\alpha D t .\right.
$$

where $\alpha$ is a constant and $D$ is the diffusion coefficient $[19,33]$.

Models of quantity of signals concentration has been developed in order to validate the process of bystander signals released by the irradiated cells. The models used the idea of diffusion, production and decay of the signals released in the medium. In 2016, Powathil et al. [31] had proposed a partial differential equation model of the signals concentration which able to describe the concentration (or strength) of the signals at specific position and specific time. In 2015, Hattori et al. [34] discussed about the cellular automaton-based model which involved the dynamic of quantity of bystander signals. The model also describes the production of DSBs is proportional to the quantities of the bystander signals.

Other than that, the concentration of bystander signals is described as the percentage or relative percentage of saturated signal rather than the signal quantity. In 2014, Lintott et al. [35] proposed a Bio-PEPA model of RIBE which the model is developed using the process of algebra method. The percentage of the signal concentration produced depends on the total number of irradiated cells and the decay rate depends on the concentration percentage. In 2012, Kundrat and Friedland [25] presented the relative percentage of saturated signal produced with respect to dose exerted towards the targeted cells. The relative percentage value of bystander signals is between zero and one which implies the strength of signal released by the irradiated cells towards bystander cells.

In addition, the simulation of bystander signals also had been analyzed using Monte Carlo method. Sasaki et al. [19] used the Monte Carlo technique in simulating the diffusion of soluble factors in cell cultures. This section concluded with suggestion of signaling model to be used in future research which is taken from Kundrat and Friedland [25],

$$
C(D)=1-\exp \left(-D / D_{C}\right)
$$

where $C$ is the relative signals concentration emitted into the medium by donor cells irradiated with dose $D$ and $D c$ is the value of characteristics and sensitivity of the donor (targeted) cells.

\section{MODELLING OF BYSTANDER EFFECTS}

Several mathematical models have been proposed about the effects of bystander phenomenon, e.g.: Brenner et al. [36] in 2001, Little et al. [37] in 2005, Xia et al. [38] and Ballarini et al. [39] in 2009, Lintott et al. [35] and Vassiliev [40] in 2014 and Hattori et al. [34] in 2015. The mechanism underlying the RIBE phenomenon is not easy to be investigated. Mathematical modelling and computational simulation offer great tools in simulating the RIBE phenomenon. Although it may not cover all the complexity of the RIBE mechanism, it could help us to understand the phenomenon.

In 2014, Lintott et al. [35] developed a Bio-PEPA model of RIBE using the process algebra method. Bio-PEPA approach is a modelling analysis specifically developed for biological applications. The model suggested that the population of bystander cells were divided into five compartments, which is healthy, infectious, apoptotic, recovered and dead. Initially, after the cells population had been ejected by specific dose of radiation; the cells that have suffered no damage from irradiation will be classed as healthy, cells that have suffered a "deadly" damage will be classed as apoptosis and cells that have suffered a "recovered" damage will be classed as infectious. The recovered and death cells initially set equally to zero. Both infectious and apoptosis cells were subjected to produce bystander signals inside the cells population. At the end of the simulation, they considered the total survival cells as initial number of cells minus death cells.

In 2015, Hattori et al. [34] investigated the mechanism of RIBE using two-dimensional cellular automaton-based model. A cellular automaton is a discrete model used to study the theoretical biology. The four components suggested in the model consists of irradiation cells, production and diffusion of bystander signals, induction of DNA DSBs by medium-mediated pathway and gap junction pathway, and the characteristic of cell-cycle phases in cell death. The concentration of bystander signals is linked to the radiation track and the absorbed dose. The production of DSBs in bystander cells also considered differently according to their pathways, either signals from medium-mediated pathway or gap junction pathway. Cell cycle phases were incorporated into their model, where each cell cycle phases have different characteristics of cell's progression. The simulation of this model is fitted to the data of a number of DSBs and cell survival from experimental data.

In 2016, Siam et al. [41] developed a mechanistic model of repair and mis-repair DNA DSBs damage. The term "mechanistic" means that the model is based on physical and chemical laws, which includes parameters with physical, chemical and biological meaning. The model described the survival of the cells after being irradiated by IR. For future research, the model will be used to describe the effect of signals emitted by the irradiated cells towards a bystander cells population. The model is explained in the following section. 


\section{The Siam et al. [41] model}

In 2016, a group of researchers have developed a repair and misrepair DNA DSBs damage model which describes how an individual cell in groups of a population evolves in time. The aim of their paper is to model the mechanism of the effect on cells directly targeted by IR using the system of ordinary differential equations (ODEs). They suggested that the number of cells can be classified into groups according to their number of DSBs initially. After the repairing process, the cells move to another group of population based on their number of DSBs and mis-repair DSBs. Since the soul of the model are based on the number of DSBs and mis-repair DSBs, according to the agreement that DSBs are formed in bystander cells, this model is suited to be applied in RIBE mechanism.

They suggested the survival cells can be determined by the initial distribution of the number of DSBs produced immediately after the IR process. Just before the time $t=0$, irradiation of dose, $D$ is given to a population of cells. At time $t=0$, the radiation process is completed and all the initial number of DSBs in each cells have been produced. The radiation dose, $D$ is incorporated into the model through the initial distribution of initial number of DSBs in each cell.

They considered $N_{k, m}$ is a group of number of cells having $k$ number of DSBs and $m$ mis-repair DSBs. The quantity evolves as follows:

$$
\begin{aligned}
& N_{k, m}(t+\Delta t)=N_{k, m}(t)-\Delta t \beta(k, m) N_{k, m}(t)-\Delta t \sum_{l=1}^{k} \gamma(k, m, l) N_{k, m}(t)+ \\
& \Delta t \sum_{j=0}^{m} \sum_{i=0}^{k_{\max }-k-m} p(k+i+j, m-j, i, j) \gamma(k+i+j, m-j, i+j) N_{k+i+j, m-j}(t) .
\end{aligned}
$$

where $\beta(k, m)$ is the death rate of cells with $k$ DSBs and $m$ mis-repair DSBs, $\gamma(k, m, l)$ is the repair rate of cells with $k$ DSBs and $m$ mis-repair DSBs repair simultaneously $l$ of them and $p(k+i+j, m-j, i, j)$ where $0<i+j<k$ and $i+j=l$ is the probability that a cell with $k+i+j$ DSBs and $m-j$ mis-repair DSBs will repair $i$ correctly and $j$ incorrectly in a unit of time.

In the biological repair process of DSBs, DSBs can be repaired more than one at a time but they only interested to the case where only one DSB repair happen at one time. From here, the model has $l=1$, which make $i+j=1$. Therefore, $\gamma(k, m, l)=\gamma(k, m, 1)$ equivalent to $\gamma(k, m)$. There is evidence that the repair rate depends on the number of repair enzymes and number of DSBs only, and then $\gamma(k, m)$ becomes $\gamma(k)$. Next, $i+j=1$ means that the model in (3) only has two situations, either $i=1, j=0$ (successful repair of one DSB) or $i=0, j=1$ (unsuccessful repair of one DSB). Hence,

$$
\begin{aligned}
N_{k, m}(t+\Delta t) & =N_{k, m}(t)-\Delta t \beta(k, m) N_{k, m}(t)-\Delta t \gamma(k) N_{k, m}(t) \\
& +\Delta t p(k+1, m, 1,0)_{\gamma}(k+1) N_{k+1, m}(t) \\
& +\Delta t p(k+1, m-1,0,1)_{Y}(k+1) N_{k+1, m-1}(t) .
\end{aligned}
$$

Since the repair process only depends on the number of DSBs, the probability of successful attempts repairing $k+1$ DSBs putted as $p(k+1,1,0)$, while unsuccessful attempts repairing $k+1$ DSBs putted as $p(k+1,0,1)$. The probability of unsuccessful attempts repairing $k+1$ DSBs modified as $1-p(k+1,1,0)$. For simplicity, they denoted the probability of a successful repair of DSBs for the cells contain $k+1$ DSBs by $p(k+1)$. By taking the limit $\Delta t \rightarrow 0$, the form of the model is written as:

$$
\begin{aligned}
\frac{d N_{k, m}}{d t}= & -\beta(k, m) N_{k, m-}(k) N_{k, m}+p(k+1)_{\gamma}(k+1) N_{k+1, m} \\
& +(1-p(k+1))_{\gamma}(k+1) N_{k+1, m-1 .}
\end{aligned}
$$

for $k=0,1,2, \ldots, k_{\max }, m=0,1,2, \ldots, k_{\max }$ with $k+m \leq k_{\max }$ and $k_{\max }$ is the maximum number of DSBs in a population of cells.

To solve the ODEs in (5), the initial condition needs to be specified. They employed Poisson distribution function with mean, $\lambda=\delta D$ in order to generate the initial number of DSBs in each cells after IR process, where $\delta$ is the radiosensitivity of targeted cells and $D$ is the radiation dose. The brief schematic description of the model (5) is shown in
Fig. 4. The blue arrow refers to successful repair, orange arrow refers to unsuccessful repair and red arrow refers to cells die.

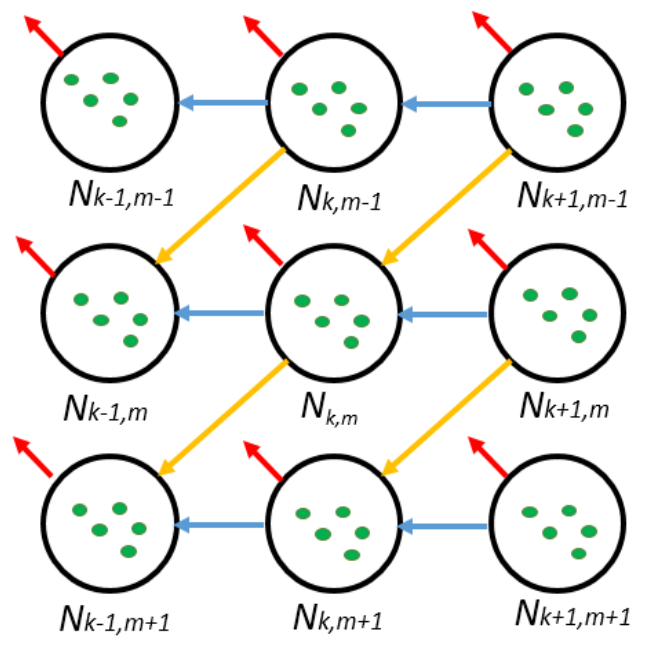

Fig. 4 Schematic representation of successful and unsuccessful repair jump into the appropriate group.

\section{SURVIVAL FRACTION MODEL}

The mathematical model proposed lead to the formation of the cell survival curve. A survival fraction $(S F)$ model represents graphically the relationship between the fraction of survival cells, $(S F)$ and the dose (D). The simulation performed by Siam et al. [41] can be fitted to the formation of survival fraction model.

If the number of the surviving cells after completed the repair process (or after a time interval investigated) denoted as $N s$, with the initial population of cells denoted as $N_{0}$, then $S F=N s / N_{0}$ is the survival fraction of a population of cells. Cell survival fraction data can be fitted by an equation of the form:

$$
S F=\exp \left(-\alpha D-\beta D^{2}\right) .
$$

where $D$ is the dose value, $\alpha$ is the probability of lethal damage due to combined of DNA DSBs and $\beta$ is the probability lethal DSBs forming from accumulated sub-lethal DSBs [42].

This paper presented two experimental data on survival fraction of direct and bystander cells obtained from published articles. Fig. 5 shows the survival fraction of AGO1522 normal human fibroblasts cells by Yang et al. [21], while Fig. 6 shows the survival fraction of human tumor cell lines UVW (glioma) by Boyd et al. [43].

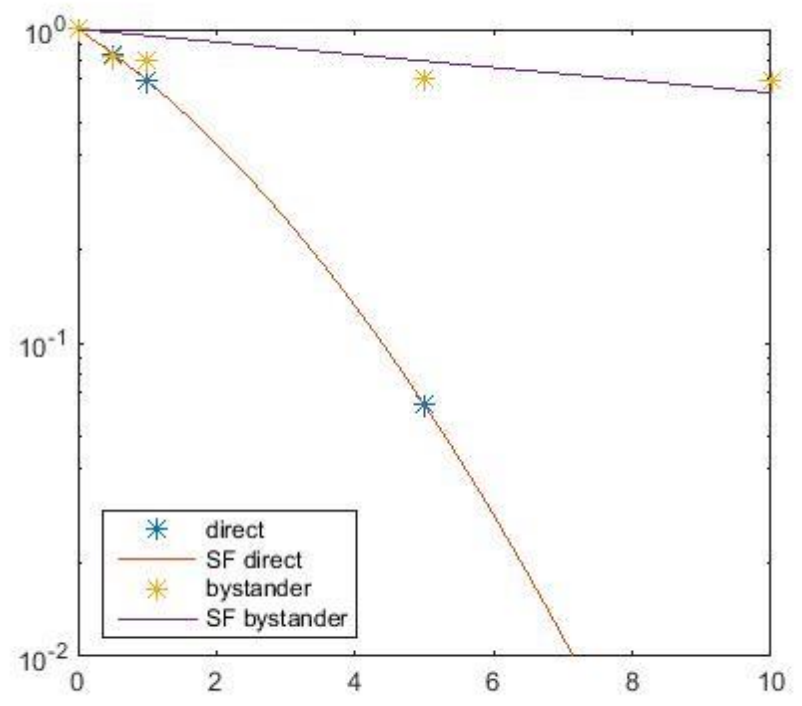

Fig. 5 Survival cells data from Yang et al. [21] and survival fraction (SF vs. $D)$. 


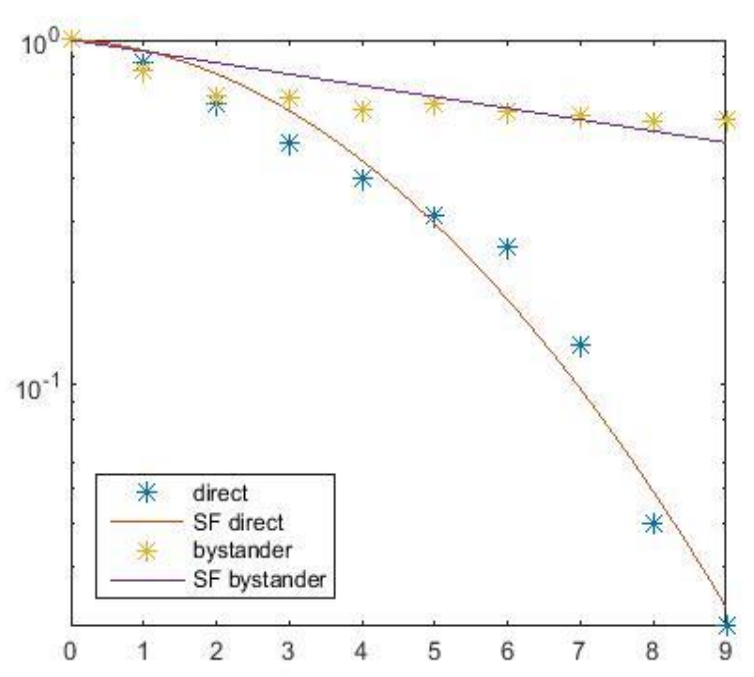

Fig. 6 Survival cells data from Boyd et al. [43] and survival fraction (SF vs. $D$ ).

The best fit to Eqn. (6) obtained from both survival fraction data are presented in Table 1. These data show that different type of cells have difference value of sentivity towards radiation dose and bystander signals. The value of sensitivity, repair rate and death rate of both data can be identified through model Eqn. (5) by using parameter estimation method [44].

Table 1 Coefficient of $\alpha$ and $\beta$ for both experimental data.

\begin{tabular}{c|c|c|c|c|c}
\hline \multicolumn{3}{c|}{ Yang et al. data } & \multicolumn{3}{c}{ Boyd et al. data } \\
\hline SF & $\alpha$ & $\beta$ & SF & $\alpha$ & $\beta$ \\
\hline Direct & 0.33437 & 0.04339 & Direct & 0.02595 & 0.04383 \\
\hline Bystander & 0.04668 & $\begin{array}{c}2.38658 \\
\times 10^{-10}\end{array}$ & Bystander & 0.07603 & $\begin{array}{c}2.40202 \\
\times 10^{-10}\end{array}$ \\
\hline
\end{tabular}

\section{RECOMMENDATIONS FOR FUTURE WORK}

As discussed thoroughly in this paper, DNA DSBs damage is formed in the bystander cells by measuring the $\mathrm{\gamma}-\mathrm{H} 2 \mathrm{AX}$ foci formation, a direct biomarker of DSBs. Due to this evidence, with in agreement towards simulation made by Hattori et al. [34], the number of DSBs formed after being in contact with danger signal molecules is proportional to the quantities of signal molecules. The probability of a bystander cells acquiring $k$ DSBs assumed to follow Poisson distribution function with mean, $\lambda=\vartheta C$ where $\vartheta$ is DSBs induction coefficient among the bystander cells and $C$ is described by Eqn. (2). This is the recommendation for initial distribution of the DNA DSBs damage in a population of bystander cells. Then, mathematical model (5) will be used to describe the repair and mis-repair DNA DSBs damage process in the bystander cells population together with modification about the repair delay process.

The next recommendations are, the simulation analysis should involve parameter estimation using the experimental data (as shown in Fig. 5 and 6). For every mathematical modelling approach towards real problem, parameter estimation is essential in order to find most suitable parameters values that reproduce experimental results according to a given set of experimental data. However, it only gives us confidence on how good the model can reproduce the set of experimental data used in the parameter estimation process. Some new predictions must be made and compare it with another experimental data that are different from the data used for parameter estimation. This is how one should investigate the model's prediction ability. Lastly, sensitivity analysis towards the model should be made. It will determine which parameters in the model have strong effects on the behaviour of the model. This will become a suggestion for radiobiology scientists in conducting their experiment method and then produce new experimental data which can be calibrated with our model.

With the above recommendations, this paper concluded here.

\section{ACKNOWLEDGEMENT}

This work was financially supported by the Universiti Teknologi Malaysia under the Fundamental Research Grant Scheme (FRGS: R.J130000.7826.4F889) and Ministry of Higher Education Malaysia (MOHE).

\section{REFERENCES}

[1] Desouky, O., Ding, N., \& Zhou, G. (2015). Targeted and non-targeted effects of ionizing radiation. Journal of Radiation Research and Applied Sciences, 8(2), 247-254.

[2] Baskar, R. (2010). Emerging role of radiation induced bystander effects: cell communications and carcinogenesis. Genome Integrity, 1(1), 13.

[3] Mothersill, C., Fernandez-Palomo, C., Fazzari, J., Smith, R., Schültke, E., Bräuer-Krisch, E., Laissue, J., Schroll, C. \& Seymour, C. (2014). Transmission of signals from rats receiving high doses of microbeam radiation to cage mates: an inter-mammal bystander effect. International Dose-Response Society, 12(1), 72-92.

[4] Marín, A., Martín, M., Liñán, O., Alvarenga, F., López, M., Fernández, L., Buchser, D. \& Cerezo, L. (2015). Bystander effects and radiotherapy. Reports of Practical Oncology \& Radiotherapy, 20(1), 12-21.

[5] Widel, M. (2016). Radiation-induced bystander effect: from in vitro studies to clinical application. International Journal of Medical Physics, Clinical Engineering and Radiation Oncology, 5(01), 1.

[6] Wang, H., Yu, K. N., Hou, J., Liu, Q., \& Han, W. (2015). Radiationinduced bystander effect: early process and rapid assessment. Cancer Letters, 356(1), 137-144.

[7] Najafi, M., Fardid, R., Hadadi, G., \& Fardid, M. (2014). The mechanisms of radiation-induced bystander effect. Journal of Biomedical Physics \& Engineering, 4(4), 163.

[8] Nikitaki, Z., Mavragani, I. V., Laskaratou, D. A., Gika, V., Moskvin, V. P., Theofilatos, K., Vougas, K., Stewart, R. D. \& Georgakilas, A. G. (2016). Systemic mechanisms and effects of ionizing radiation: a new 'old' paradigm of how the bystanders and distant can become the players. In Seminars in Cancer Biology, 37, 77-95.

[9] Chevalier, F., Hamdi, D. H., Saintigny, Y., \& Lefaix, J. L. (2015). Proteomic overview and perspectives of the radiation-induced bystander effects. Mutation Research/Reviews in Mutation Research, 763, 280-293.

[10] Sprung, C. N., Ivashkevich, A., Forrester, H. B., Redon, C. E., Georgakilas, A., \& Martin, O. A. (2015). Oxidative DNA damage caused by inflammation may link to stress-induced non-targeted effects. Cancer Letters, 356(1), 72-81.

[11] Olobatuyi, O., de Vries, G., \& Hillen, T. (2016). A reaction-diffusion model for radiation-induced bystander effects. Journal of Mathematical Biology, 1-32.

[12] Mothersill, C., \& Seymour, C. (2013). Radiation-induced bystander effects and stress-induced mutagenesis. Stress-Induced Mutagenesis Springer New York, 199-222.

[13] Han, W., \& Yu, K. N. (2010). Ionizing radiation, DNA double strand break and mutation. Advances in Genetics Research, 4, 197-210.

[14] Havaki, S., Kotsinas, A., Chronopoulos, E., Kletsas, D., Georgakilas, A., \& Gorgoulis, V. G. (2015). The role of oxidative DNA damage in radiation induced bystander effect. Cancer letters, 356(1), 43-51.

[15] Mole, R. H. (1953). Whole body irradiation - radiobiology or medicine? The British Journal of Radiology, 26(305), 234-241.

[16] Nagasawa, H., \& Little, J. B. (1992). Induction of sister chromatid exchanges by extremely low doses of $\alpha$-particles. Cancer Research, 52(22), 6394-6396.

[17] Sokolov, M. V., Smilenov, L. B., Hall, E. J., Panyutin, I. G., Bonner, W. M., \& Sedelnikova, O. A. (2005). Ionizing radiation induces DNA doublestrand breaks in bystander primary human fibroblasts. Oncogene, 24(49), 7257-7265.

[18] Hu, B., Han, W., Wu, L., Feng, H., Liu, X., Zhang, L., Xu, A., Hei, T. K. \& Yu, Z. (2005). In situ visualization of DSBs to assess the extranuclear/extracellular effects induced by low-dose $\alpha$-particle irradiation. Radiation Research, 164(3), 286-291.

[19] Sasaki, K., Wakui, K., Tsutsumi, K., Itoh, A., \& Date, H. (2012). A simulation study of the radiation-induced bystander effect: modeling with stochastically defined signal reemission. Computational and Mathematical Methods in medicine. 
[20] Seymour, C. B., \& Mothersill, C. (2000). Relative contribution of bystander and targeted cell killing to the low-dose region of the radiation dose-response curve. Radiation Research, 153(5), 508-511.

[21] Yang, H., Asaad, N., \& Held, K. D. (2005). Medium-mediated intercellular communication is involved in bystander responses of X-ray-irradiated normal human fibroblasts. Oncogene, 24(12), 2096-2103.

[22] Hatzi, V. I., Laskaratou, D. A., Mavragani, I. V., Nikitaki, Z., Mangelis, A., Panayiotidis, M. I., Pantelias, G. E., Terzoudi, G. I. \& Georgakilas, A. G. (2015). Non-targeted radiation effects in vivo: a critical glance of the future in radiobiology. Cancer letters, 356(1), 34-42.

[23] Hall, E. J., \& Giaccia, A. J. (2006). Radiobiology for the Radiologist Lippincott Williams \& Wilkins.

[24] Wondergem, J. (2010). Radiation biology: a handbook for teachers and students. International Atomic Energy Agency.

[25] Kundrát, P., \& Friedland, W. (2012). Non-linear response of cells to signals leads to revised characteristics of bystander effects inferred from their modelling. International Journal of Radiation Biology, 88(10), 743750 .

[26] Liu, Y., Kobayashi, A., Maeda, T., Fu, Q., Oikawa, M., Yang, G., Konishi, T., Uchihori, Y., Hei, T. K. \& Wang, Y. (2015). Target irradiation induced bystander effects between stem-like and non stem-like cancer cells. Mutation Research/Fundamental and Molecular Mechanisms of Mutagenesis, 773, 43-47.

[27] Sedelnikova, O. A., Nakamura, A., Kovalchuk, O., Koturbash, I., Mitchell, S. A., Marino, S. A., Brenner, D. J. \& Bonner, W. M. (2007). DNA doublestrand breaks form in bystander cells after microbeam irradiation of threedimensional human tissue models. Cancer Research, 67(9), 4295-4302.

[28] Stein, G. S., \& Pardee, A. B. (2004). Cell cycle and growth control: biomolecular regulation and cancer. John Wiley \& Sons.

[29] Prise, K. M., Schettino, G., Folkard, M., \& Held, K. D. (2005). New insights on cell death from radiation exposure. The Lancet Oncology, 6(7), 520-528.

[30] Lara, P. C., López-Peñalver, J. J., de Araújo Farias, V., Ruiz-Ruiz, M. C., Oliver, F. J., \& de Almodóvar, J. M. R. (2015). Direct and bystander radiation effects: a biophysical model and clinical perspectives. Cancer Letters, 356(1), 5-16.

[31] Powathil, G. G., Munro, A. J., Chaplain, M. A., \& Swat, M. (2016) Bystander effects and their implications for clinical radiation therapy: insights from multiscale in silico experiments. Journal of Theoretical Biology, 401, 1-14.

[32] Ojima, M., Furutani, A., Ban, N., \& Kai, M. (2010). Persistence of DNA double-strand breaks in normal human cells induced by radiation-induced bystander effect. Radiation Research, 175(1), 90-96.

[33] Facoetti, A., Mariotti, L., Ballarini, F., Bertolotti, A., Nano, R., Pasi, F., Ranza, E. \& Ottolenghi, A. (2009). Experimental and theoretical analysis of cytokine release for the study of radiation-induced bystander effect. International Journal of Radiation Biology, 85(8), 690-699.

[34] Hattori, Y., Yokoya, A., \& Watanabe, R. (2015). Cellular automaton-based model for radiation-induced bystander effects. BMC Systems Biology, 9(1), 90 .

[35] Lintott, R., McMahon, S., Prise, K., Addie-Lagorio, C., \& Shankland, C. (2014). Using process algebra to model radiation induced bystander effects. In International Conference on Computational Methods in Systems Biology, 196-210.

[36] Brenner, D. J., Little, J. B., \& Sachs, R. K. (2001). The bystander effect in radiation oncogenesis: II. A quantitative model. Radiation Research, 155(3), 402-408

[37] Little, M. P., Filipe, J. A. N., Prise, K. M., Folkard, M., \& Belyakov, O. V. (2005). A model for radiation-induced bystander effects, with allowance for spatial position and the effects of cell turnover. Journal of Theoretical Biology, 232(3), 329-338.

[38] Xia, J., Liu, L., Xue, J., Wang, Y., \& Wu, L. (2009). Modeling of radiationinduced bystander effect using Monte Carlo methods. Nuclear Instruments and Methods in Physics Research Section B: Beam Interactions with Materials and Atoms, 267(6), 1015-1018.

[39] Ballarini, F., Facoetti, A., Mariotti, L., Nano, R., \& Ottolenghi, A. (2009). Cellular communication and "non-targeted effects": modelling approaches. Advances in Space Research, 44(8), 917-925.

[40] Vassiliev, O. N. (2014). A model of radiation-induced bystander effect based on an analogy with ferromagnets. Application to modelling tissue response in a uniform field. Physica A: Statistical Mechanics and its Applications, 416: 242-251.

[41] Siam, F. M., Grinfeld, M., Bahar, A., Rahman, H. A., Ahmad, H., \& Johar, F. (2016). A mechanistic model of high dose irradiation damage. Mathematics and Computers in Simulation.

[42] Ebert, M. A., Suchowerska, N., Jackson, M. A., \& McKenzie, D. R. (2010). A mathematical framework for separating the direct and bystander components of cellular radiation response. Acta Oncologica, 49(8), 1334 1343.

[43] Boyd, M., Ross, S. C., Dorrens, J., Fullerton, N. E., Tan, K. W., Zalutsky, M. R., \& Mairs, R. J. (2006). Radiation-induced biologic bystander effect elicited in vitro by targeted radiopharmaceuticals labeled with $\alpha-, \beta-$, and Auger electron-emitting radionuclides. Journal of Nuclear Medicine, 47(6), 1007-1015.

[44] Siam, F. M., Kamal, M. H. A., \& Johar, F. (2016). Parameter Estimation For A Mechanistic Model Of High Dose Irradiation Damage Using Nelder-Mead Simplex Method And Genetic Algorithm. Jurnal Teknologi, 78(12-2), 87-92. 